\title{
Figures of merit of thermoelectric and bolometric thermal radiation sensors
}

\author{
U. Dillner, E. Kessler, and H.-G. Meyer \\ Institute of Photonic Technology, Albert-Einstein-Str. 9, 07745 Jena, Germany \\ Correspondence to: U. Dillner (ulrich.dillner@ipht-jena.de)
}

Received: 15 February 2013 - Revised: 13 May 2013 - Accepted: 15 May 2013 - Published: 21 June 2013

\begin{abstract}
Figures of merit condensing the performance parameters of radiation sensors such as responsivity, noise equivalent power, and time constant in a single number can be useful for rating the performance of a particular sensor in comparison to other ones or to fundamental performance limits. The classification system and the figures of merit of radiation sensors introduced by R. C. Jones are revisited for thermal radiation sensors with the focus on thermopiles and bolometers. As a result it is stated that radiation thermopiles and bolometers should be classified differently: type III detectors for thermopiles vs. type II detectors for bolometers. Modified figures of merit are suggested and relations between them given. The figures of merit are applied in an overview on state-of-the-art thermopiles and bolometers operated at room temperature.
\end{abstract}

\section{Introduction}

Thermal radiation sensors belong to the class of thermal sensors (van Herwaarden and van Oudheusden, 1994; Budzier and Gerlach, 2011). They are based on two signal transduction stages: radiation to thermal and thermal to electrical. In the first stage, the input radiation power is converted into heat by an absorber, which creates or changes a temperature gradient in a thermal isolation structure. In the second stage, this intermediate signal is converted into the electrical output signal using a temperature (difference) transducer. The thermal sensor principle constitutes the fundamental difference between thermal and photonic radiation sensors (e.g. photoconductors) based on quantum detection and results in such typical features of thermal radiation sensors as uncooled operation and broadband response over the infrared spectrum enabled by appropriate volume absorbers.

Radiation sensors can be characterized by several measured quantities; among them are responsivity, time constant, and noise equivalent power, which are important parameters to assess the suitability of a given radiation sensor for a specific application. For a comparison of various radiation sensors it is expedient to condense these parameters preferably in a single number serving as a figure of merit, which can help the potential user of these sensors to rate the perfor- mance of a particular sensor in comparison to other ones or to fundamental performance limits due to physical principles set by, e.g. temperature fluctuations and background radiation. R. C. Jones pioneered this topic comprising the ultimate sensitivity of radiation sensors (Jones, 1947) and their classification (Jones, 1949a) as well as appropriate figures of merit (Jones, 1949b) and the introduction of the specific detectivity $D^{*}$ (Jones, 1957). The unit of measurement of $D^{*}$ was later named after him $\left(\mathrm{cmHz}^{1 / 2} \mathrm{~W}^{-1}=\right.$ Jones $)$ in recognition of his work.

The purpose of the present paper is to revisit the topic for thermal radiation sensors. We will focus on two very common thermal radiation sensors: the bolometer and the radiation thermocouple or thermopile, the latter terming a serial connection of a number of thermocouples. The bolometer uses the thermoresistive effect, i.e. the temperature dependence of an electric resistor, for temperature sensing, while the thermopile applies the thermoelectric or Seebeck effect in a thermocouple to implement a temperature difference transducer (for respective reviews of bolometers and thermopiles for infrared detection see, e.g. Richards, 1994, and Graf et al., 2007).

Our paper is organized as follows: the basic theory of thermal radiation sensors will be set forth in Sects. 2 and 3. Summarizing these sections, Fig. 1 describes in a pictorial way 
Thermal radiation detector: 2-step signal generation

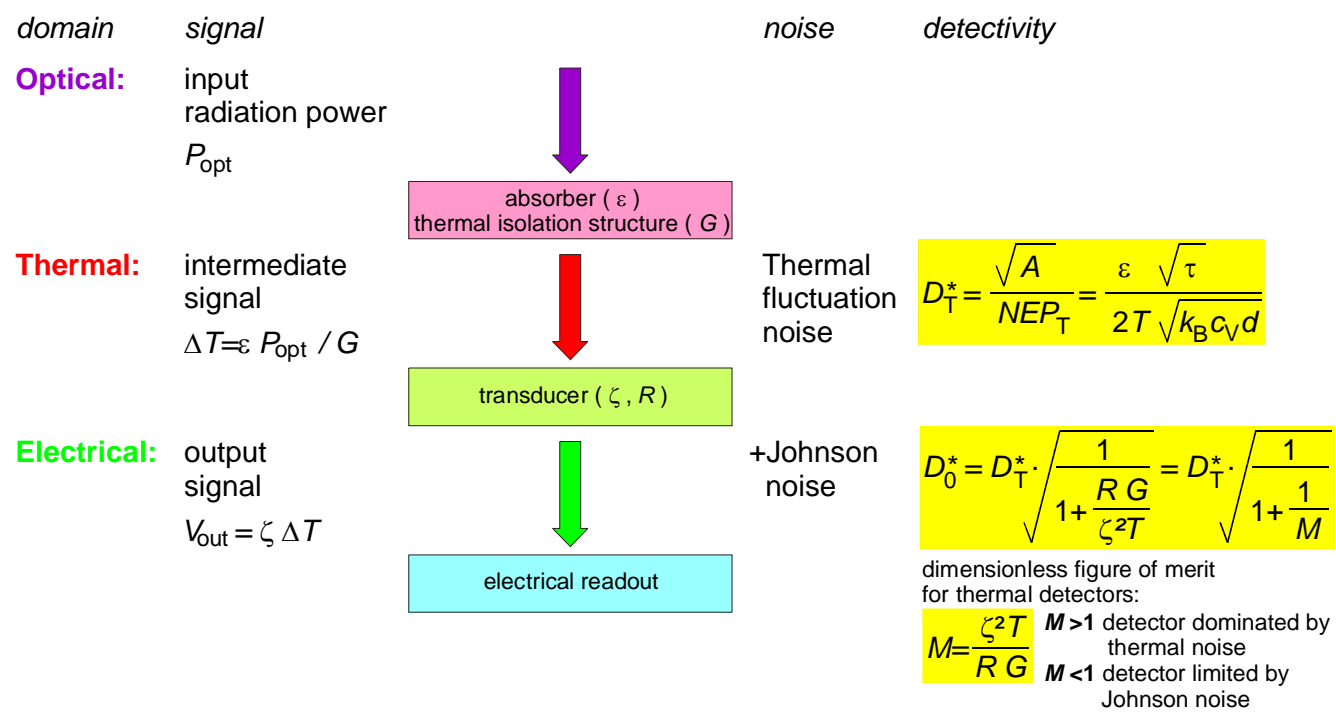

Figure 1. Illustration of the sensing principle of thermal radiation detectors including the signal generation, the main sources of noise, and the resulting specific detectivity.

Table 1. Summary of the figures of merit for bolometers and thermocouples/thermopiles.

\begin{tabular}{llll}
\hline Quantity & Bolometer & Thermocouple(pile) & Ratio (BO/TC) \\
\hline$n$ & 2 & 3 & \\
\hline$k_{n}^{\prime}\left(\mathrm{m} / \mathrm{Ws}^{n / 2}\right)$ & $\begin{array}{l}k_{2}^{\prime}=D^{*} / \sqrt{\tau} \\
\text { cf. Eq. }(38)\end{array}$ & $\begin{array}{l}k_{3}^{\prime}=D^{*} / \tau \\
\text { cf. Eq. }(38)\end{array}$ & $k_{2}^{\prime} / k_{3}^{\prime}=\sqrt{\tau}$ \\
\hline$M_{\mathrm{nH}}$ (dimensionless) & $\begin{array}{l}M_{2 \mathrm{H}}=k_{2}^{\prime} / k_{2 \mathrm{H}}^{\prime} \\
\text { cf. Eq. }(44)\end{array}$ & $\begin{array}{l}M_{3 \mathrm{H}}=k_{3}^{\prime} / k_{3 \mathrm{H}}^{\prime} \\
\text { cf. Eq. }(46)\end{array}$ & $M_{2 \mathrm{H}} / M_{3 \mathrm{H}}=\sqrt{\tau / \tau_{\mathrm{H}}}$ \\
\hline$M_{n}$ (dimensionless) & $\begin{array}{l}M_{2}=k_{2}^{\prime} / k_{2 \mathrm{~T}}^{\prime}\left(c_{\mathrm{A}}\right) \\
\text { cf. Eqs. }(43),(39)\end{array}$ & $\begin{array}{l}M_{3}=k_{3}^{\prime} / k_{3 \mathrm{~J}}^{\prime}\left(c_{\mathrm{A}}\right) \\
\text { cf. Eqs. }(50),(51),(40)\end{array}$ & $M_{2} / M_{3}=\sqrt{\tau / \tau_{\mathrm{R}}\left(c_{\mathrm{A}}\right)}$ \\
\hline
\end{tabular}

the general idea and the key formulae concerning signal generation, noise, and specific detectivity of these sensors. In Sect. 4, Jones' statement that both thermopiles and bolometers can be classified as type II detectors (Jones, 1949a) will be reviewed and modified figures of merit suggested, especially for thermopiles. Table 1 provides a summary of these figures of merit, which will then be applied in Sect. 5 to quantify their performance in an overview of state-of-the-art thermal radiation sensors based on room-temperature-operated thermopiles and bolometers.

\section{Parameters of thermal radiation sensors}

\subsection{Heat capacitance, conductance, and transfer coefficient}

The thermal isolation structure, which is necessary to generate the temperature difference $\Delta T$ as the intermediate signal of the thermal sensor, is characterized by its heat capacitance
$C$ and heat conductance $G$. If we assume that the thermal isolation structure is a membrane of thickness $d$ and a size essentially comprising the receiving area $A$ of the sensor, which results in a volume $V=A d$, then the heat capacitance can be readily calculated from the corresponding specific quantities, i.e. the volumetric heat capacity $c_{\mathrm{V}}$ and the heat capacity per unit area $c_{\mathrm{A}}=c_{\mathrm{V}} d$,

$C=c_{\mathrm{V}} V=c_{\mathrm{A}} A$.

The heat conductance results from the temperature difference $\Delta T$ generated by the heat load $P$ applied at the membrane according to

$G=P / \Delta T$.

Corresponding to the membrane geometry, it is useful to relate the thermal conductance to the receiving area by a heat transfer coefficient

$H=G / A$. 
The total heat transfer comprises three components: by radiation $\left(H_{\mathrm{R}}\right)$, by conduction of the functional layers forming the temperature transducer $\left(H_{\mathrm{C}}\right)$, and by parasitic heat flows $\left(H_{\mathrm{P}}\right)$ originating, e.g. from a surrounding gas atmosphere or any layers of the thermal isolation structure other than the functional layers considered above, hence

$H=\sum_{i} H_{i}=H_{\mathrm{R}}+H_{\mathrm{C}}+H_{\mathrm{P}}$

(index $i=R, C, P$ ). The heat transfer by radiation sets a minimum for the total heat transfer. Its heat transfer coefficient can be calculated from Stefan's law assuming $\Delta T \ll T$, which results in

$H_{\mathrm{R}}=4 \varepsilon \sigma_{\mathrm{SB}} T^{3}$

(cf. Eq. 3.6 in Jones, 1947). Here $\varepsilon$ is the absorptivity or emissivity of the receiving area, $\sigma_{\mathrm{SB}}$ the Stefan-Boltzmann constant, and $T$ the operational temperature. Equation (5) yields $H_{\mathrm{R}}=6.12 \mathrm{~W} \mathrm{~m}^{-2} \mathrm{~K}^{-1}$ at $T=300 \mathrm{~K}$ and $\varepsilon=1$. The heat transfer by conduction of the functional layers is proportional to their thermal conductivity $\kappa$, hence

$H_{\mathrm{C}}=\kappa / g$,

where $g$ is a geometric factor expressed in length units.

\subsection{Responsivity and time constant}

The responsivity $S$ of a thermal sensor is given by

$S=\frac{S_{0}}{1+j \cdot 2 \pi f \tau}, \quad S_{0}=\frac{\varepsilon|\varsigma|}{G}$,

where $S_{0}$ denotes the dc $(f=0)$ responsivity, $j$ the imaginary unit, $f$ the operational frequency, and $\tau$ the thermal time constant, which reflects the dynamic behaviour of the sensor,

$\tau=C / G=c_{\mathrm{A}} / H$

Based on the analysis presented in Eq. (4), the reciprocal time constant is analogously written as

$\frac{1}{\tau}=\sum_{i} \frac{1}{\tau_{i}}=\frac{1}{\tau_{\mathrm{R}}}+\frac{1}{\tau_{\mathrm{C}}}+\frac{1}{\tau_{\mathrm{P}}}, \quad \tau_{i}=\frac{c_{\mathrm{A}}}{H_{i}}$.

The thermometric transduction coefficient $\zeta$ (unit of measurement $\mathrm{V} \mathrm{K}^{-1}$ ) indicates the strength of the transduction from the thermal to the electrical domain by the transducer. Considering the radiation thermocouple (TC), the transduction coefficient is equal to its Seebeck coefficient $\gamma$. A bolometer (BO), however, needs a bias current causing a voltage drop $U$ to generate the signal voltage:

$\varsigma^{(\mathrm{TC})}=\gamma, \quad \varsigma^{(\mathrm{BO})}=\alpha U$.

Here $\alpha=(1 / R)(\mathrm{d} R / \mathrm{d} T)$ is the temperature coefficient of the electrical resistance $R$ of the bolometer. Thus, the essential difference regarding the transduction coefficients of these alternative thermal radiation sensors is that $\zeta^{(\mathrm{TC})}$ contrary to $\zeta^{(\mathrm{BO})}$ depends on a material parameter only. For comparison of the bias-dependent properties of bolometers of different size and resistance, it is advisable to refer to their biasinduced temperature differences. The bias voltage can be expressed in terms of the Joule heating power $P$ or, with Eq. (2), the bias-induced temperature difference $\Delta T$ as

$U=\sqrt{R P}=\sqrt{R G \Delta T}$.

Using Eq. (8), one obtains a relation between $U$ and $\tau$, which reads

$U=\sqrt{R C \Delta T / \tau}$

\subsection{Noise equivalent power and detectivity}

The noise equivalent power $N$ of a radiation sensor is the incident radiation power yielding a signal-to-noise ratio of unity at the sensor's output. Usually, the squared noise equivalent power per unit bandwidth $\Delta f$,

$\mathrm{NEP}^{2}=N^{2} / \Delta f$,

is considered, which results in the unit of measurement $\mathrm{W} \mathrm{Hz}^{-1 / 2}$ for NEP. Actually, Eq. (13) applies to the optical NEP, which has to be distinguished from the electrical NEP referring to an ideal (blackbody) emissivity $\varepsilon=1$ according to the relation $\mathrm{NEP}_{\mathrm{el}}=\varepsilon \cdot \mathrm{NEP}$. The bandwidth is limited by the dynamic behaviour of the sensor, i.e.

$\Delta f=\int_{0}^{\infty}\left|\frac{S}{S_{0}}\right|^{2} \mathrm{~d} f=\int_{0}^{\infty} \frac{\mathrm{d} f}{1+(2 \pi f \tau)^{2}}=\frac{1}{4 \tau}$.

With the maximum bandwidth from Eq. (14), $\mathrm{NEP}^{2}=4 N^{2} \tau$ is deduced for the minimum NEP from Eq. (13). The detectivity $D$ is simply the reciprocal of the NEP, while the specific detectivity $D^{*}$ is defined by $D^{*}=A^{1 / 2} D$ (Jones, 1957), hence

$D^{*}=\sqrt{A \Delta f} / N=\sqrt{A} / \mathrm{NEP}$.

The total NEP of a thermal sensor comprises at least two principal components caused by the temperature fluctuation noise $\left(\mathrm{NEP}_{\mathrm{T}}\right)$ of the thermal isolation structure (also termed phonon or thermal noise) and by the Johnson noise $\left(\mathrm{NEP}_{\mathrm{J}}\right)$ of the temperature transducer, which amounts to

$\mathrm{NEP}^{2}=\mathrm{NEP}_{\mathrm{T}}^{2}+\mathrm{NEP}_{\mathrm{J}}^{2}$

The following analysis will be restricted to these fundamental components, neglecting additional noise sources such as, e.g. $1 / f$ noise. 


\section{Analysis of the principal noise components}

\subsection{Temperature fluctuation noise}

The mean square temperature fluctuation depends on the heat capacitance and is given by

$\overline{\Delta T^{2}}=k_{\mathrm{B}} T^{2} / C$,

where $k_{\mathrm{B}}$ denotes Boltzmann's constant, cf. Eq. (2.4) in Jones (1947). By analogy with the squared Eq. (2), the relation

$G^{2}=\frac{\mathrm{NEP}_{\mathrm{Tel}}^{2} \Delta f}{\overline{\Delta T^{2}}}, \quad \mathrm{NEP}_{\mathrm{Tel}}^{2}=\left(\varepsilon \cdot \mathrm{NEP}_{\mathrm{T}}\right)^{2}$

can be established, where $\mathrm{NEP}_{\text {Tel }}$ is the electrical NEP due to temperature fluctuations. Using Eqs. (8), (14), and (17) Eq. (18) can be rewritten (Mather, 1982) as

$\mathrm{NEP}_{\mathrm{Tel}}^{2}=4 k_{\mathrm{B}} T^{2} G$.

The corresponding specific detectivity bounded by temperature fluctuations is readily calculated from Eqs. (3), (15), and (19) to be

$D_{\mathrm{Tel}}^{*}=\frac{1}{2 T \sqrt{k_{\mathrm{B}} H}}=\frac{1}{2 T} \sqrt{\frac{\tau}{k_{\mathrm{B}} c_{\mathrm{A}}}}$.

\subsection{Johnson noise}

From the Johnson noise power spectral density $4 k_{\mathrm{B}} T R$ of the transducer's electrical resistance $R$, the NEP due to Johnson noise is given by

$\mathrm{NEP}_{\mathrm{J}}^{2}=4 k_{\mathrm{B}} T R /|S|^{2}$.

Equation (21) can be related to Eq. (19), which results in

$\left(\frac{\mathrm{NEP}_{\mathrm{J}}}{\mathrm{NEP}_{\mathrm{T}}}\right)^{2}=\frac{R}{R_{\mathrm{D}}} \cdot\left[1+(2 \pi f \tau)^{2}\right]$

by applying Eq. (7) and introducing the dynamic resistance $R_{\mathrm{D}}=\zeta^{2} T / G$, cf. Smith et al. (1968). Since operation at $f>0$ increases the Johnson noise, we will hereafter consider its dc minimum, designated by the subscript " 0 ", $\mathrm{NEP}_{\mathrm{J} 0}=$ $\left(R / R_{\mathrm{D}}\right)^{1 / 2} \mathrm{NEP}_{\mathrm{T}}$. The resistance ratio $R_{\mathrm{D}} / R$ is also known as the dimensionless figure of merit $M$ of a thermal sensor,

$M=\varsigma^{2} T /(R G)$,

hence

$\mathrm{NEP}_{\mathrm{J} 0}^{2}=\mathrm{NEP}_{\mathrm{T}}^{2} / M$.

$M \ll 1$ stands for prevailing Johnson noise, while $M \gg$ 1 means that temperature fluctuation noise is dominating. Again, based on the analysis by Eq. (4) and substituting $H_{i}$ by $\tau_{i}$, cf. Eq. (9), the reciprocal dimensionless figure of merit can be written as

$\frac{1}{M}=\sum_{i} \frac{1}{M_{i}}=\frac{1}{M_{\mathrm{R}}}+\frac{1}{M_{\mathrm{C}}}+\frac{1}{M_{\mathrm{P}}}, \quad M_{i}=\frac{\tau_{i}}{\mu}$.

Here

$\mu=\frac{\tau}{M}=\frac{R C}{S^{2} T}$

can be interpreted as the time constant in the case that both Johnson and temperature fluctuation noise have an equal share in the total noise $(M=1)$. The corresponding specific detectivity bounded by Johnson noise at dc operation is

$D_{\mathrm{J} 0}^{*}=D_{\mathrm{T}}^{*} \sqrt{M}=D_{\mathrm{T}}^{*} \sqrt{\tau / \mu}, \quad D_{\mathrm{T}}^{*}=\varepsilon \cdot D_{\mathrm{Tel}}^{*}$.

The different expressions of the transduction coefficient $\zeta$ of bolometers and thermocouples, cf. Eq. (10), translate into differing relations for $\mu$ and $M=\tau / \mu$, respectively. Due to the dependence of the bias voltage $U$ on $\tau$, cf. Eq. (12), $\mu^{(\mathrm{BO})}$ is proportional to $\tau$ while $M^{(\mathrm{BO})}$ does not depend on $\tau$. Using Eqs. (10) and (12),

$\mu^{(\mathrm{BO})}=\frac{\tau}{M^{(\mathrm{BO})}}, \quad M^{(\mathrm{BO})}=\alpha^{2} T \Delta T$

is deduced from Eq. (26). Concerning thermocouples, however, $M^{(\mathrm{TC})}$ is proportional to $\tau$ while $\mu^{(\mathrm{TC})}$ does not depend on $\tau$ in a reversal of the respective results for bolometers:

$\mu^{(\mathrm{TC})}=\frac{c_{\mathrm{A}} R A}{\gamma^{2} T}, \quad M^{(\mathrm{TC})}=\frac{\tau}{\mu^{(\mathrm{TC})}}$.

Alternatively to Eq. (29), $\mu^{(\mathrm{TC})}$ can be expressed by the relation $\mu^{(\mathrm{TC})}=\tau_{\mathrm{C}} / M_{\mathrm{C}}^{(\mathrm{TC})}$, cf. Eq. (25) for $i=C . M_{\mathrm{C}}^{(\mathrm{TC})}$ is identical to the dimensionless thermoelectric figure of merit $Z T$, which is the crucial material parameter for increasing the thermoelectric conversion efficiency. This parameter is related to the material properties of the thermocouple transducer according to the formula (Nolas et al., 2001)

$Z T=\left(\frac{\gamma_{p}-\gamma_{n}}{\sqrt{\kappa_{p} \rho_{p}}+\sqrt{\kappa_{n} \rho_{n}}}\right)^{2} T$.

Here $\gamma_{m}$ with $m=n, p$ is the absolute Seebeck coefficient of the $m$ type thermocouple leg combining into $\gamma=\gamma_{p}-\gamma_{n}, \kappa_{m}$ is its thermal conductivity, and $\rho_{m}$ its electrical resistivity. Note that $Z T$ referring to the heat transfer by conduction of the functional layers has to be distinguished from the general dimensionless figure of merit $M$ referring to the total heat transfer. In fact, $M^{(\mathrm{TC})}<M_{\mathrm{C}}^{(\mathrm{TC})}=Z T$ holds due to parasitic and radiative heat losses in accordance with Eq. (25). Equation (20) as well as Eqs. (28) and (29) allow for specifying the detectivity formula presented by Eq. (27). Thus,

$D_{\mathrm{J} 0}^{*(\mathrm{BO})}=\frac{\varepsilon|\alpha|}{2} \sqrt{\frac{\Delta T}{c_{\mathrm{A}} k_{\mathrm{B}} T}} \sqrt{\tau}$ 


\section{Detectivity: radiation thermocouples vs. bolometers}
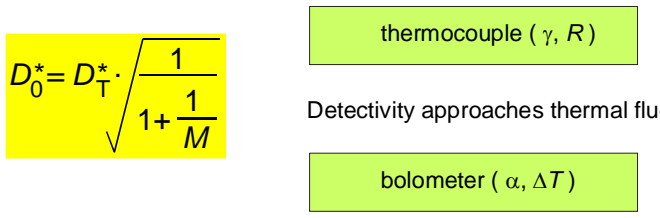
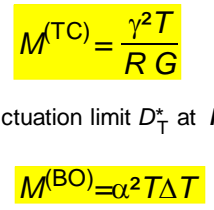

large Seebeck coefficient $\gamma$

good thermal isolation, i.e.

low thermal conductance $G$

low electrical resistance $R$

large temperature coefficient

of resistance $\alpha$

large bias temperature $\Delta T$

Figure 2. Options for raising the specific detectivity of thermocouples and bolometers.

and

$D_{\mathrm{J} 0}^{*(\mathrm{TC})}=\frac{\varepsilon \gamma}{2 c_{\mathrm{A}}} \frac{\tau}{\sqrt{k_{\mathrm{B}} T R A}}$

are obtained.

\subsection{Total noise}

The total noise in the dc limit is given by

$\mathrm{NEP}_{0}^{2}=\mathrm{NEP}_{\mathrm{T}}^{2}(1+1 / M)$

in accordance with Eqs. (16) and (24). The corresponding specific detectivity reads

$D_{0}^{*}=D_{\mathrm{T}}^{*} \sqrt{\frac{M}{1+M}}=D_{\mathrm{T}}^{*} \sqrt{\frac{\tau}{\mu+\tau}}$.

Specifying this equation, again for bolometers and thermocouples individually, yields

$D_{0}^{*(\mathrm{BO})}=\frac{\varepsilon}{2} \frac{\sqrt{\tau}}{\sqrt{c_{\mathrm{A}} k_{\mathrm{B}} T\left(\frac{1}{\alpha^{2} \Delta T}+T\right)}}$

and

$D_{0}^{*(\mathrm{TC})}=\frac{\varepsilon \gamma}{2} \frac{\tau}{\sqrt{c_{\mathrm{A}} k_{\mathrm{B}} T\left(c_{\mathrm{A}} R A+\gamma^{2} T \tau\right)}}$.

Evidently, Eqs. (32) and (36) related to thermocouples also apply to thermopiles.

Figure 1 outlines the basic theory of thermal radiation sensors presented in Sects. 2 and 3. The differences between thermocouples and bolometers as to their dimensionless figure of merit, and hence their specific detectivity depicted in Fig. 2, are crucial regarding their different classification in the subsequent Sect. 4.

\section{Sensor classification and figures of merit}

\subsection{Classification system}

R. C. Jones introduced a classification system for radiation detectors by relating their noise equivalent power $N$ to the receiving area $A$ and time constant $\tau$ according to

$N(A, \tau)=\left(1 / k_{n}\right) \sqrt{A / \tau^{n}}$, cf. Eq. (9.2) in Jones (1949a), where $k_{n}$ is a constant, which is independent of $A$ and $\tau$. The exponent $n$ is the number determining the classification as a type $n$ detector. Evidently, since for a given area and time constant it is desired to make $N$ as small as possible, $k_{n}$ is a figure of merit for the type $n$ detector (Jones, 1949b). Concerning bolometers and thermopiles, both were classified as type II detectors by Jones (1949a). Applying Eqs. (13), (14), and (15), Eq. (37) can be rewritten in terms of the specific detectivity $D^{*}$ to be

$D^{*}(\tau)=k_{n}^{\prime} \sqrt{\tau^{n-1}}$,

where $k_{n}^{\prime}=k_{n} / 2$ is the modified figure of merit related to $D^{*}$ for the type $n$ detector. While equivalent to the one based on the noise equivalent power, the classification based on the dependence of the specific detectivity on the time constant is probably a particularly concise way to classify a radiation detector. Due to Eq. (38) the specific detectivity of type I detectors is independent of $\tau$, while being proportional to $\tau$ for type III detectors. Type II detectors lie in between with a proportionality to $\tau^{1 / 2}$.

Comparing Eq. (38) with Eq. (20) we find that thermal radiation sensors dominated by temperature fluctuation noise $(M \gg 1)$ are type II detectors with a specific detectivity,

$D_{\mathrm{Tel}}^{*}(\tau)=k_{2 \mathrm{~T}}^{\prime} \sqrt{\tau}, \quad k_{2 \mathrm{~T}}^{\prime}\left(c_{\mathrm{A}}\right)=\frac{1}{2 T \sqrt{k_{\mathrm{B}} c_{\mathrm{A}}}}$.

\subsection{Thermodynamic and Havens' limit}

It is useful to refer $k_{n}$ and $k_{n}^{\prime}$ to some limiting values, thus replacing these figures having rather inconvenient dimensional units $\left(\mathrm{m} \mathrm{W}^{-1} \mathrm{~s}^{-n / 2}\right)$ by dimensionless figures of merit for type $n$ detectors with unity as their value at the respective limit. The radiative heat exchange quantified by $H_{\min }=H_{\mathrm{R}}$, cf. Eq. (5), inevitably establishes a fundamental thermodynamic limit corresponding to a lower bound on the noise equivalent power and an upper bound on the time constant

$\tau_{\max }=\tau_{\mathrm{R}}\left(c_{\mathrm{A}}\right)=c_{\mathrm{A}} / H_{\mathrm{R}}$

in accordance with Eq. (9) as well as on the specific detectivity, which is calculated to be

$D_{\text {max }}^{*}=D_{\mathrm{Tel}}^{*}\left(\tau_{\max }\right)=k_{1 \mathrm{~T}}^{\prime}, \quad k_{1 \mathrm{~T}}^{\prime}=\frac{1}{4 \sqrt{k_{\mathrm{B}} \sigma_{\mathrm{SB}} T^{5}}}$ 
by applying Eqs. (39) and (40) and substituting $H_{\mathrm{R}}$ at $\varepsilon=1$ using Eq. (5). Here $k_{1 \mathrm{~T}}^{\prime}$ is the limiting value referring to type I detectors. Hence, the dimensionless figure of merit $M_{1}$ for a type I detector, which was proposed by Jones, cf. Eq. (2.4) in Jones (1949b), reads

$M_{1}=k_{1}^{\prime} / k_{1 \mathrm{~T}}^{\prime}$.

We readily calculate $k_{1 \mathrm{~T}}^{\prime}=1.81 \times 10^{8} \mathrm{~m} \mathrm{~W}^{-1} \mathrm{~s}^{-1 / 2}$ at $T=$ $300 \mathrm{~K}$ corresponding to a maximum specific detectivity of $1.81 \times 10^{10} \mathrm{cmHz}^{1 / 2} \mathrm{~W}^{-1}$.

Analogously to Eq. (42), the dimensionless figure of merit $M_{2}$ for a type II detector can be written as

$M_{2}=k_{2}^{\prime} / k_{2 \mathrm{~T}}^{\prime}\left(c_{\mathrm{A}}\right)$.

The limiting value $k_{2 \mathrm{~T}}^{\prime}$ referring to type II detectors and correlated with the physical limit due to the temperature fluctuation noise, cf. Eq. (39), depends not only on $T$ but also on the heat capacity per unit area $c_{\mathrm{A}}$. This is an essential drawback in regard of the practical usability of $M_{2}$ since data concerning $c_{\mathrm{A}}$ are commonly unavailable to the user contrary to $D^{*}$ and $\tau$ data. $k_{2}^{\prime}$ can be expressed in terms of the minimum detectable energy related to the square root of the receiving area $Q=N \tau / A^{1 / 2}$ (unit of measurement $\mathrm{Ws} \mathrm{m}^{-1}$ ). Rewriting Eq. (37) for $n=2$ yields $Q=1 / k_{2}=1 /\left(2 k_{2}^{\prime}\right)$. Hence, the limiting value $k_{2 \mathrm{~T}}^{\prime}$ corresponds to a lower bound $Q_{\min }=1 /\left(2 k_{2 \mathrm{~T}}^{\prime}\right)$ $\propto c_{\mathrm{A}}^{1 / 2}$. In principle, $c_{\mathrm{A}}=c_{\mathrm{V}} d$ and hence $Q_{\min }$ can be arbitrarily low. In practice, however, there are threshold values concerning the thickness $d$ as well as the volumetric heat capacity $c_{\mathrm{V}}$ of the thermal isolation structure. Havens (1946) made an engineering estimate of the minimum detectable energy of thermal radiation sensors at room temperature based on the state of the art in the middle of the 20th century. Known as Havens' limit, it was in no way intended to be a fundamental limit, and set a value $Q_{\mathrm{H}}=3 \times 10^{-9} \mathrm{Ws} \mathrm{m}^{-1}$, which translates into $k_{2 \mathrm{H}}^{\prime}=1.67 \times 10^{8} \mathrm{~m} \mathrm{~W}^{-1} \mathrm{~s}^{-1}$. Using this value instead of the general limiting value $k_{2 \mathrm{~T}}^{\prime}\left(c_{\mathrm{A}}\right)$ we get, as a special case of Eq. (43), another dimensionless figure of merit $M_{2 \mathrm{H}}$ for a type II detector, which reads

$M_{2 \mathrm{H}}=k_{2}^{\prime} / k_{2 \mathrm{H}}^{\prime}$.

This figure of merit was originally proposed by Jones, cf. Eq. (2.6) in Jones (1949b). The heat capacity per unit area corresponding to Havens' limit is calculated from $k_{2 \mathrm{H}}^{\prime}=$ $k_{2 \mathrm{~T}}^{\prime}\left(c_{\mathrm{AH}}\right)$ to be $c_{\mathrm{AH}}=7.24 \mathrm{Ws} \mathrm{m}^{-2} \mathrm{~K}^{-1}$. With Eq. (40) the maximum time constant of a sensor obeying Havens' limit is $\tau_{\mathrm{H}}=\tau_{\mathrm{R}}\left(c_{\mathrm{AH}}\right)=1.18 \mathrm{~s}$. Havens' limit can also be expressed by use of the specific detectivity

$D_{2 \mathrm{H}}^{*}(\tau)=k_{2 \mathrm{H}}^{\prime} \sqrt{\tau}$

\subsection{Classification of thermopiles and bolometers}

The classification is based on Eq. (38). Due to the proportionality $D^{*} \propto \tau^{1 / 2}$, cf. Eq. (35), bolometers are type II detectors regardless of whether temperature fluctuation or Johnson noise is dominating. Hence, the above figures of merit $M_{2}$ and $M_{2 \mathrm{H}}$ are applicable. Thermocouples and thermopiles, however, exhibit a linear dependence $D^{*} \propto \tau$ in the case of prevailing Johnson noise, cf. Eq. (32). Hence, they should be classified differently as type III detectors in that case. Moreover, $M^{(\mathrm{TC})}<Z T=1$ holds in practice since, in spite of all efforts in thermoelectric materials research, no material showing a dimensionless thermoelectric figure of merit significantly greater than $Z T \sim 1$ has been found to date (Venkatasubramanian et al., 2001). Given the fact that typically $M^{(\mathrm{TC})} \ll Z T$ due to primarily parasitic heat flows, it can be assumed that Johnson noise is prevailing for thermopiles in virtually all cases. On this view, their different classification compared with bolometers is generally applicable. Advanced thermoelectric materials based on superlattice structures with $Z T$ up to 2.4 (Venkatasubramanian et al., 2001) have not been employed in radiation thermopiles yet, first of all owing to their delicate preparation. If, in future, improved radiation thermopiles based on such materials can be fabricated, while avoiding major parasitic losses, then the intermediate case between type II and type III, cf. Eq. (36), could gain some practical significance.

Obviously, the physical reason for the different classification of thermocouples and thermopiles on the one hand and bolometers on the other hand is the difference in the transduction coefficients, cf. Eq. (10). While the thermocouple has a constant $\zeta_{\mathrm{TC}}$, the bolometer exhibits a $\zeta_{\mathrm{BO}}$ proportional to the bias voltage $U$, which in turn is proportional to $1 / \tau^{1 / 2}$, cf. Eq. (12). If, as an example, the time constant is halved by any modification, e.g. the use of a better conducting filling gas to enhance $H_{\mathrm{P}}$, this will result in a corresponding halving of the specific detectivity of a radiation thermopile. Considering a bolometer, however, the halving of the time constant enables an increase of the bias voltage by a factor square root of 2 to maintain the bias-induced temperature difference, which partially compensates the reduction of the specific detectivity resulting in its decrease only by a factor square root of 2 instead of its halving.

For thermopiles as type III detectors certainly the figures of merit $M_{2}$ and $M_{2 \mathrm{H}}$ are not applicable. Instead, by analogy with Havens' limit, it would seem natural to replace $M_{2 \mathrm{H}}$ by a figure of merit $M_{3 \mathrm{H}}$,

$M_{3 \mathrm{H}}=k_{3}^{\prime} / k_{3 \mathrm{H}}^{\prime}$.

The corresponding specific detectivity replacing Eq. (45) is

$D_{3 \mathrm{H}}^{*}(\tau)=k_{3 \mathrm{H}}^{\prime} \tau$,

where

$D_{3 \mathrm{H}}^{*}\left(\tau_{\mathrm{H}}\right)=D_{2 \mathrm{H}}^{*}\left(\tau_{\mathrm{H}}\right)=D_{\max }^{*}$.

From Eq. (48) it can be deduced that

$k_{3 \mathrm{H}}^{\prime}=k_{2 \mathrm{H}}^{\prime} / \sqrt{\tau_{\mathrm{H}}}$, 


\section{Comparison to experiment}

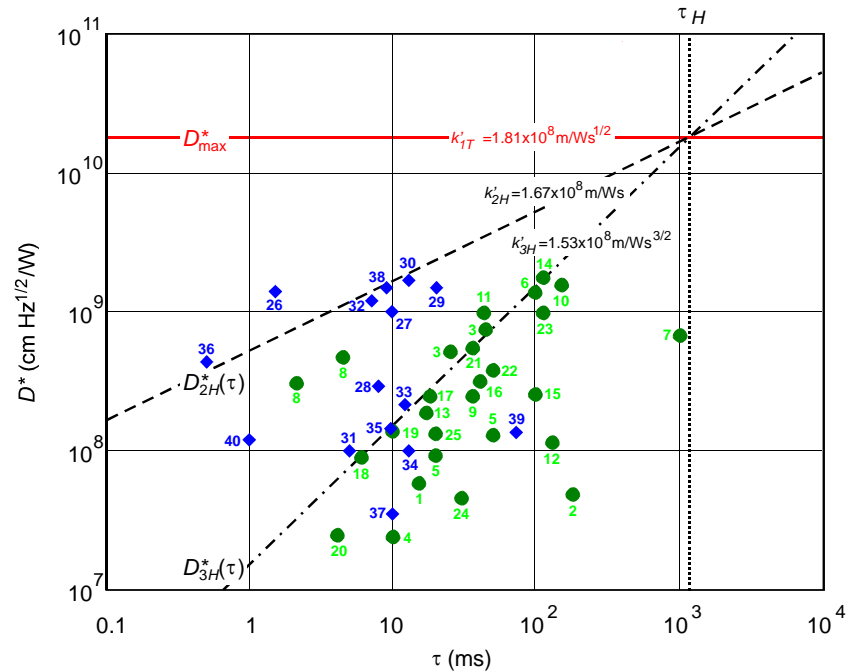

Figure 3. Parameters $D^{*}$ and $\tau$ of a selection of commercial as well as non-commercial thermoelectric $(\bullet)$ and bolometric $(\bullet)$ radiation sensors and sensor arrays operated at room temperature. For the tag numbers cf. Tables 3 and $4 . D_{\max }^{*}(\tau)$ is the specific detectivity due to the thermodynamic limit (solid line), $D_{2 \mathrm{H}}^{*}(\tau)$ the one due to Havens' limit related to type II detectors (dashed line) and $D_{3 \mathrm{H}}^{*}(\tau)$ the one due to its type III detector analogue (dot-dashed line). $\tau_{\mathrm{H}}$ is the maximum time constant within Havens' limit (dotted line).

resulting in $k_{3 \mathrm{H}}^{\prime}=1.53 \times 10^{8} \mathrm{~m} \mathrm{~W}^{-1} \mathrm{~s}^{-3 / 2}$. As its counterpart $k_{2 \mathrm{H}}^{\prime}$ the limiting value $k_{3 \mathrm{H}}^{\prime}$ does not correspond to a fundamental limit. In other words, contrary to $M_{1}$, the figures of merit $M_{2 \mathrm{H}}$ and $M_{3 \mathrm{H}}$ can be greater than 1 . However, though Havens' estimate is more than $60 \mathrm{yr}$ old, figures of $M_{2 \mathrm{H}}$ and $M_{3 \mathrm{H}}$ above unity indicating an outstanding performance are still the exception for bolometers or thermopiles operated at room temperature. Generalizing the concept leading to $M_{3 \mathrm{H}}$, a figure of merit $M_{3}$ can be introduced by analogy with $M_{2}$, cf. Eq. (43), as

$M_{3}=k_{3}^{\prime} / k_{3 \mathrm{~J}}^{\prime}\left(c_{\mathrm{A}}\right)$.

Here $k_{3 \mathrm{~J}}^{\prime}$ is the limiting value referring to type III detectors given analogously to Eq. (49) by

$k_{3 \mathrm{~J}}^{\prime}\left(c_{\mathrm{A}}\right)=k_{2 \mathrm{~T}}^{\prime}\left(c_{\mathrm{A}}\right) / \sqrt{\tau_{\mathrm{R}}\left(c_{\mathrm{A}}\right)}$.

Again $k_{3 \mathrm{~J}}^{\prime}$ depends not only on $T$ but also on $c_{\mathrm{A}}$, whereby $k_{3 \mathrm{H}}^{\prime}=k_{3 \mathrm{~J}}^{\prime}\left(c_{\mathrm{AH}}\right)$ applies analogously to $k_{2 \mathrm{H}}^{\prime}=k_{2 \mathrm{~T}}^{\prime}\left(c_{\mathrm{AH}}\right)$. Contrary to $M_{2}$, which is correlated with the fundamental temperature fluctuation noise limit, but similar to $M_{2 \mathrm{H}}$ and $M_{3 \mathrm{H}}$ the figure of merit $M_{3}$ does not refer to a fundamental limit.

For clarity reasons all figures of merit relating to bolometers and thermopiles are compiled again in Table 1. Moreover, these figures can be related to the dimensionless figure of merit $M=R_{\mathrm{D}} / R$, cf. Eq. (23), and its radiative component $M_{\mathrm{R}}$, cf. Eq. (25). These relations are summarized in Table 2. Considering these relations it becomes clear how
Table 2. Summary of the relations between the figures of merit quoted in Table 1 and the dimensionless figure of merit $M$ and its radiative component $M_{\mathrm{R}}$.

\begin{tabular}{lll}
\hline Bolometer & Thermocouple(pile) & Ratio (BO/TC) \\
\hline$k_{2}^{\prime}=k_{2 \mathrm{~T}}^{\prime} \sqrt{\frac{M}{1+M}}$ & $k_{3}^{\prime}=\frac{k_{2 \mathrm{~T}}^{\prime}}{\sqrt{\mu+\tau}}=k_{2 \mathrm{~T}}^{\prime} \sqrt{\frac{M}{\tau(1+M)}}$ & $\frac{k_{2}^{\prime}}{k_{3}^{\prime}}=\sqrt{\tau}$ \\
\hline$M_{2 \mathrm{H}}=\sqrt{\frac{c_{\mathrm{AH}}}{c_{\mathrm{A}}} \frac{M}{1+M}}$ & $M_{3 \mathrm{H}}=\frac{c_{\mathrm{AH}}}{c_{\mathrm{A}}} \sqrt{\frac{M_{\mathrm{R}}}{1+M}}$ & $\frac{M_{2 \mathrm{H}}}{M_{3 \mathrm{H}}}=\sqrt{\frac{c_{\mathrm{A}}}{c_{\mathrm{AH}}} \frac{M}{M_{\mathrm{R}}}}$ \\
\hline$M_{2}=\sqrt{\frac{M}{1+M}}$ & $M_{3}=\sqrt{\frac{M_{\mathrm{R}}}{1+M}}$ & $\frac{M_{2}}{M_{3}}=\sqrt{\frac{M}{M_{\mathrm{R}}}}$ \\
\hline
\end{tabular}

Comparison to experiment: Thermocouples

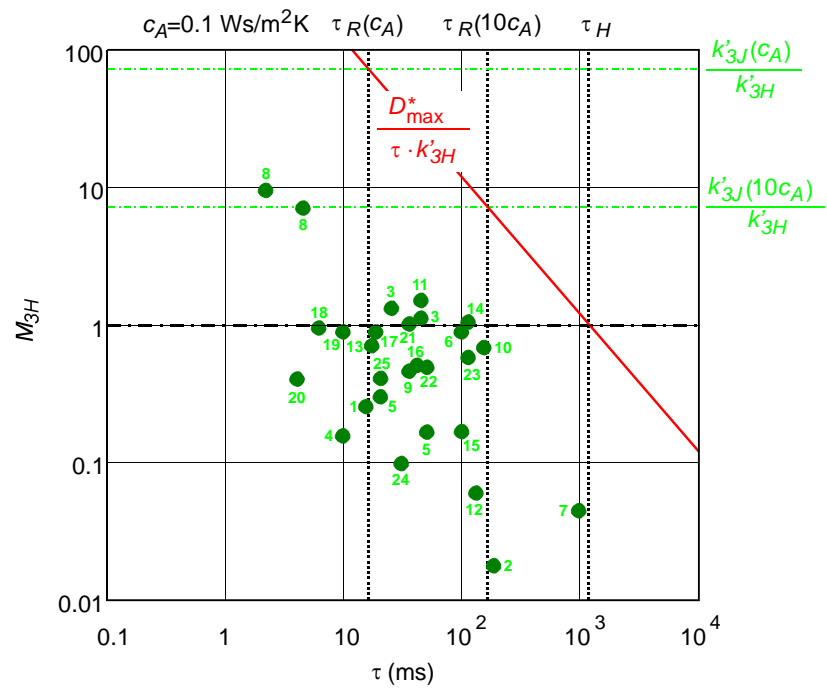

Figure 4. Dimensionless figure of merit $M_{3 \mathrm{H}}$ measuring $D^{*} / \tau$ of the thermoelectric radiation sensors plotted in Fig. 3 in units of $k_{3 \mathrm{H}}^{\prime}$. For the limiting value $k_{3 \mathrm{~J}}^{\prime}\left(c_{\mathrm{A}}\right) \mathrm{cf}$. Eq. (51).

the figures of merit $k_{2}^{\prime}$ and $k_{3}^{\prime}$ as well as their dimensionless equivalents $M_{2 \mathrm{H}}$ and $M_{3 \mathrm{H}}$ can be pushed to their limits set by thermal fluctuation noise as represented by $k_{2 \mathrm{~T}}^{\prime}$. For bolometers, $M^{(\mathrm{BO})}$ and hence the temperature coefficient of resistance $\alpha$ and the bias temperature $\Delta T$ have to be maximized. For thermocouples, on the other hand, $\mu^{(\mathrm{TC})}$ has to be minimized, which means that the ratio $\gamma^{2} / R$ (also known as power factor since a high Seebeck coefficient combined with a low resistance is advantageous) has to be maximized, whereas the heat capacitance $C$ has to be minimized. Thus, employing better transducer materials showing higher values of $\alpha$ and $\gamma^{2} / R$ is the first option for improvement. Employing better technologies aiming at realizing very thin (low $C$ ) but mechanically and thermally (high $\Delta T$ ) stable structures is the second option. At the thermal fluctuation limit, however, the only way to further improve these figures of merit at room temperature is a reduction of the heat capacity per unit area $c_{\mathrm{A}}=c_{\mathrm{V}} d$ as expressed by Eq. (39), which again calls for improved technologies needed to enable very thin but still functional thermal isolation structures. 
Table 3. Dimensionless figure of merit $M_{3 \mathrm{H}}$ and references of the tag numbers of the thermoelectric radiation sensors, cf. Figs. 3 and 4.

\begin{tabular}{|c|c|c|}
\hline Tag number & $M_{3 \mathrm{H}}$ & Reference \\
\hline 1 & 0.26 & Lahiji and Wise (1982) \\
\hline 2 & 0.018 & Sarro et al. (1988) \\
\hline 3 & 1.141 .36 & Völklein et al. (1991) \\
\hline 4 & 0.16 & Lenggenhager et al. (1993) \\
\hline 5 & 0.170 .30 & Schieferdecker et al. (1995) \\
\hline 6 & 0.91 & Foote et al. (1998) \\
\hline 7 & 0.046 & Müller et al. (2000) \\
\hline 8 & 7.129 .63 & Foote et al. (2003) \\
\hline 9 & 0.47 & Dillner et al. (2004) \\
\hline 10 & 0.70 & Dillner et al. (2006) \\
\hline 11 & 1.52 & Hirota et al. (2007) \\
\hline 12 & 0.06 & Wang et al. (2010) \\
\hline 13 & 0.73 & Chen (2012) \\
\hline 14 & 1.07 & Haenschke et al. (2012) \\
\hline 15 & 0.17 & Thermopile 3M Datasheet, Dexter Research Center Inc., www.dexterresearch.com \\
\hline 16 & 0.52 & Thermopile DR46 Datasheet, Dexter Research Center Inc., www.dexterresearch.com \\
\hline 17 & 0.91 & Thermopile S25 Datasheet, Dexter Research Center Inc., www.dexterresearch.com \\
\hline 18 & 0.98 & Thermopile HTS A11 Datasheet, Heimann Sensor GmbH, www.heimannsensor.com \\
\hline 19 & 0.91 & Thermopile HTS A21 Datasheet, Heimann Sensor GmbH, www.heimannsensor.com \\
\hline 20 & 0.41 & Thermopile Array HTPA 32x31 Datasheet, Heimann Sensor GmbH, www.heimannsensor.com \\
\hline 21 & 1.03 & Thermopile TS-80 Datasheet, IPHT, www.ipht-jena.de \\
\hline 22 & 0.51 & Thermopile TS-144 Datasheet, IPHT, www.ipht-jena.de \\
\hline 23 & 0.59 & Thermopile Array TPL640Xe Datasheet, Micro-Hybrid Electronic GmbH, http://www.micro-hybrid.de \\
\hline 24 & 0.10 & Thermopile MLX90247 Datasheet, Melexis Microelectronic Integrated Systems, www.melexis.com \\
\hline 25 & 0.42 & Thermopile T11262-01 Datasheet, Hamamatsu Photonics K.K., www.hamamatsu.com \\
\hline
\end{tabular}

\section{Overview on room-temperature-operated thermopiles and bolometers}

This section is intended to provide an overview, which makes no claim to being complete but is for illustration purposes only, on thermoelectric and bolometric radiation sensors in the framework of the above figures of merit. Spanning the latest 3 decades, the diagrams presented in Figs. 3 to 5 include a selection of commercial as well as non-commercial sensors and sensor arrays operated at room temperature. In the diagrams, a particular sensor is tagged by a number referenced in Table 3 for thermopiles and in Table 4 for bolometers. A double occurrence of a reference number in a diagram represents two different sensors qualified in one and the same reference. If the detectivity was not reported explicitly in a reference, it was recalculated from related $S, R$, and $A$ data or from the published noise equivalent temperature difference (NETD) achieved by the thermal imaging setup with a focal plane array (FPA) consisting of corresponding bolometer or thermopile pixels. Similarly, the time constant, if not explicitly given, was recalculated from the bandwidth.

Figure 3 presents an overall picture of both $D^{*}$ and $\tau$ of all sensors considered and enables a comparison of the data among each other and with the thermodynamic limit as well as with Havens' limit for type II detectors and its analogue for type III detectors. The values of the specific detectivity of the sensors span almost 2 orders of magnitude between $2.4 \times 10^{7} \mathrm{cmHz}^{1 / 2} \mathrm{~W}^{-1}$ and $1.8 \times 10^{9} \mathrm{cmHz}^{1 / 2} \mathrm{~W}^{-1}$, while the time constants include more than 3 orders of magnitude between $0.5 \mathrm{~ms}$ and $1 \mathrm{~s}$. Figure 4 shows the dimensionless figure of merit $M_{3 \mathrm{H}}$ of the thermoelectric radiation sensors with values reaching from 0.018 to 9.63. The dimensionless figure of merit $M_{2 \mathrm{H}}$ of the bolometric radiation sensors with values reaching from 0.021 to 2.17 is presented in Fig. 5. Notwithstanding that $M_{3 \mathrm{H}}$ as well as $M_{2 \mathrm{H}}$ can exceed unity at best, both the thermoelectric and the bolometric sensors are mostly Johnson noise limited corresponding to $M<1$, cf. Eq. (24).

The separate presentation of thermopiles and bolometers in Figs. 4 and 5, respectively, complies with their different classification and enables a comparison of their data with the related limiting values $k_{3 \mathrm{~J}}^{\prime}$ and $k_{2 \mathrm{~T}}^{\prime}$. Both these limiting values depend on the heat capacity per unit area. They increase with decreasing $c_{\mathrm{A}}$ so that, in the end, $c_{\mathrm{A}}=c_{\mathrm{V}} d$ is the key limiting parameter and should be as low as possible. The $M_{3 \mathrm{H}}$ and $M_{2 \mathrm{H}}$ value of a particular sensor allows for the calculation of an upper bound on $c_{\mathrm{A}}$ of the sensor concerned without knowing $c_{\mathrm{A}}$ itself by use of the formulae $c_{\mathrm{A}} \leq c_{\mathrm{AH}} / M_{3 \mathrm{H}}$ and $c_{\mathrm{A}} \leq c_{\mathrm{AH}} / M_{2 \mathrm{H}}^{2}$, respectively. These inequalities are easily deduced from the relations concerning $M_{3 \mathrm{H}}$ and $M_{2 \mathrm{H}}$ listed in Table 2 . As a guide, the limits correlated with $c_{\mathrm{A}}=0.1 \mathrm{Ws} \mathrm{m}^{-2} \mathrm{~K}^{-1}$ and its tenfold value $1 \mathrm{Ws} \mathrm{m}^{-2} \mathrm{~K}^{-1}$ are explicitly indicated in the graphs of Figs. 4 
Table 4. Dimensionless figure of merit $M_{2 \mathrm{H}}$ and references of the tag numbers of the bolometric radiation sensors, cf. Figs. 3 and 5.

\begin{tabular}{cll}
\hline Tag number & $M_{2 \mathrm{H}}$ & Reference \\
\hline 26 & 2.17 & Schnelle et al. (1984) \\
27 & 0.60 & Wood (1993) \\
28 & 0.20 & Tanaka et al. (1996) \\
29 & 0.64 & Radford et al. (1998) \\
30 & 0.88 & Dong et al. (2002) \\
31 & 0.085 & Zintu et al. (2002) \\
32 & 0.86 & Tissot et al. (2005) \\
33 & 0.12 & Yue et al. (2006) \\
34 & 0.053 & Liu et al. (2007) \\
35 & 0.087 & Saxena et al. (2008) \\
36 & 1.15 & Karanth et al. (2009) \\
37 & 0.021 & Kumar and Butler (2009) \\
38 & 0.95 & Tissot et al. (2010) \\
39 & 0.031 & Wang and Li (2010) \\
40 & 0.23 & Vera-Reveles et al. (2011) \\
\hline
\end{tabular}

and 5 together with the corresponding upper bounds on the time constant, cf. Eq. (40). Thus, considering Fig. 4, it can be concluded that, e.g. the heat capacity per unit area of the thermopile sensor showing the highest values of $M_{3 \mathrm{H}}$ might be considerably below the level of $1 \mathrm{Ws} \mathrm{m}^{-2} \mathrm{~K}^{-1}$ indicated in the graph. Similarly, considering Fig. 5, the heat capacity per unit area of the bolometer showing the highest value of $M_{2 \mathrm{H}}$ is concluded to be again clearly below that level. Assuming a volumetric heat capacity $c_{\mathrm{V}}=10^{6} \mathrm{Ws} \mathrm{m}^{-3} \mathrm{~K}^{-1}$ as an estimate of its order of magnitude, the resulting thickness $d$ would consequently be of the order of $100 \mathrm{~nm}$ in either case.

\section{Conclusions}

Since bolometers and radiation thermopiles show differences in their dependence of the specific detectivity on the time constant $\left(D^{*} \propto \tau^{1 / 2}\right.$ vs. $\left.D^{*} \propto \tau\right)$ they should be classified differently (type II vs. type III detectors). As a consequence of this different behaviour, the ratio $k_{2}^{\prime}=D^{*} / \sqrt{\tau}$ is an appropriate figure of merit for rating the performance of a particular bolometer-based thermal radiation sensor in comparison to other ones, while $k_{3}^{\prime}=D^{*} / \tau$ is its equivalent appropriate to thermopile radiation sensors. To create dimensionless figures of merit for convenience it is expedient to relate the dimensioned figures $k_{2}^{\prime}$ and $k_{3}^{\prime}$ to some suitable reference values, e.g. $k_{2 \mathrm{H}}^{\prime}=1.67 \times 10^{8} \mathrm{~m} \mathrm{~W}^{-1} \mathrm{~s}^{-1}$ and $k_{3 \mathrm{H}}^{\prime}=1.53 \times 10^{8} \mathrm{~m} \mathrm{~W}^{-1} \mathrm{~s}^{-3 / 2}$, which correspond to Havens' limit representing no fundamental limit but an early engineering estimate.

Edited by: U. Schmid

Reviewed by: two anonymous referees

\section{Comparison to experiment: Bolometers}

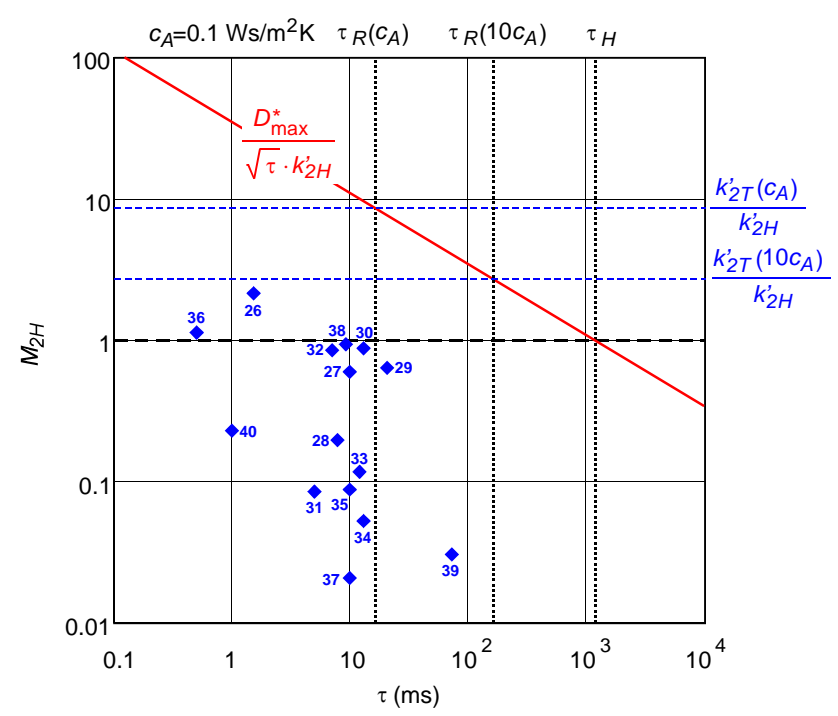

Figure 5. Dimensionless figure of merit $M_{2 \mathrm{H}}$ measuring $D^{*} / \sqrt{\tau}$ of the bolometric radiation sensors plotted in Fig. 3 in units of $k_{2 \mathrm{H}}^{\prime}$. For the limiting value $k_{2 \mathrm{~T}}^{\prime}\left(c_{\mathrm{A}}\right) \mathrm{cf}$. Eq. (39).

\section{References}

Budzier, H. and Gerlach, G.: Thermal infrared sensors, Wiley, Chichester, 2011.

Chen, C.-N.: Fully quantitative characterization of CMOS-MEMS polysilicon/titanium thermopile infrared sensors, Sensor. Actuat. B-Chem., 161, 892-900, 2012.

Dillner, U., Baier, V., Kessler, E., Müller, J., Berger, A., Behrendt, D., and Preller, H.-A.: A high sensitivity single-chip 4-element thermoelectric infrared sensor, in: Proceedings of the 8th International Conference for Infrared Sensors and Systems, Nuremberg, Germany, 25-27 May 2004, 149-153, 2004.

Dillner, U., Kessler, E., Baier, V., Berger, A., Eick, T., Behrendt, D., and Urban, H.: A 64-pixel linear thermopile array chip designed for vacuum environment, Proceedings of the 9th International Conference for Infrared Sensors and Systems, Nuremberg, Germany, 30 May-1 June 2006, 295-300, 2006.

Dong, L., Yue, R.-F., and Liu, L.-T.: An uncooled microbolometer infrared detector based on polycrystalline silicon germanium thin film, Int. J. Nonlin. Sci. Num., 3, 303-306, 2002.

Foote, M. C., Jones, E. W., and Caillat, T.: Uncooled thermopile infrared detector linear arrays with detectivity greater than $10^{9} \mathrm{cmHz}^{1 / 2} / \mathrm{W}$, IEEE T. Electron Dev., 1896-1902, 1998.

Foote, M. C., Kenyon, M., Krueger, T. R., McCann, T. A., Chacon, R., Jones, E. W., Dickie, M. R., Schofield, J. T., McCleese, D. J., Gaalema, S., and Hu, W.: Thermopile detector arrays for space science applications, Proceedings of the International Workshop on Thermal Detectors for Space Based Planetary, Solar, and Earth Science Applications, Adelphi, USA, 19-20 June 2003, 2, 16-20, 2003.

Graf, A., Arndt, M., Sauer, M., and Gerlach, G.: Review of micromachined thermopiles for infrared detection, Meas. Sci. Technol., 18, R59-R75, 2007. 
Haenschke, F., Kessler, E., Dillner, U., Ihring, A., Schinkel, U., and Meyer, H.-G.: New high detectivity linear array for analytical measurement in the room temperature range, Proceedings of SPIE 8353, Infrared Technology and Applications XXXVIII, Baltimore, USA, 23-27 April 2012, 83531L1-6, 2012.

Havens, R.: Theoretical comparison of heat detectors, J. Opt. Soc. Am., 36, p. 355, 1946.

Hirota, M., Nakajima, Y., Saito, M., and Uchiyama, M.: $120 \times 90$ element thermoelectric infrared focal plane array with precisely patterned Au-black absorber, Sensor. Actuat. A-Phys., 135, 146151, 2007.

Jones R. C.: The ultimate sensitivity of radiation detectors, J. Opt. Soc. Am., 37, 879-890, 1947.

Jones R. C.: A new classification system for radiation detectors, J. Opt. Soc. Am., 39, 327-343, 1949a.

Jones, R. C.: Factors of merit for radiation detectors, J. Opt. Soc. Am., 39, 344-356, 1949b.

Jones, R. C.: Method of rating the performance of photoconductive cells, Proc. IRIS, 2, 9-12, 1957.

Karanth, S., Sumesh, M. A., Shobha, V., Ganesh Shanbhogue, H., and Nagendra, C. L.: Infrared detectors based on thin film thermistor of ternary Mn-Ni-Co-O on micro-machined thermal isolation structure, Sensor. Actuat. A-Phys., 153, 69-75, 2009.

Kumar, S. and Butler, D. P.: Infrared sensing with self-supporting YBCO uncooled IR microbolometer array integrated with onchip CCBDI readout circuit, IEEE Sens. J., 9, 411-418, 2009.

Lahiji, G. R. and Wise, K. D.: A batch-fabricated silicon thermopile infrared detector, IEEE T. Electron Dev., 29, 14-22, 1982.

Lenggenhager, R., Baltes, H., and Elbel, T.: Thermoelectric infrared sensors in CMOS technology, Sensor. Actuat. A-Phys., 37-38, 216-220, 1993.

Liu, X.-M., Fang, H.-J., and Liu, L.-T.: Study on new structure uncooled a-Si microbolometer for infrared detection, Microelectron. J., 38, 735-739, 2007.

Mather, J. C.: Bolometer noise: nonequilibrium theory, Appl. Optics, 21, 1125-1129, 1982.

Müller, J. E., Kessler, E., Dillner, U., Ratz, P., Stock, K. D., and Metzdorf, J.: Large sized high sensitive thin-film thermopile as radiometric detector standard, Proceedings of the Symposium on Microtechnology in Metrology and Metrology in Microsystems, Delft, The Netherlands, 31 August-1 September 2000, 147-148, 2000.

Nolas, G. S., Sharp, J., and Goldsmid, H. J.: Thermoelectrics - basic principles and new materials developments, Springer, Berlin, Heidelberg and New York, 2001.

Radford, W., Wyles, R., Wyles, J., Varesi, J., Ray, M., Murphy, D., Kennedy, A., Finch, A., Moody, E., Cheung, F., Coda, R., and Baur, S.: Sensitivity improvements in uncooled microbolometer FPAs, Report approved for public release, Raytheon Infrared Center of Excellence, Goleta, www.dtic.mil/dtic/tr/fulltext/u2/ a399216.pdf, 1998.

Richards, P. L.: Bolometers for infrared and millimeter waves, J. Appl. Phys., 76, 1-24, 1994.

Sarro, P. M., Yashiro, H., van Herwaarden, A. W., and Middelhoek, S.: An integrated thermal infrared sensing array, Sensor. Actuator., 14, 191-201, 1988.

Saxena, R. S., Bhan, R. K., Jalwania, C. R., Rana, P. S., and Lomash, S. K.: Characterization of area arrays of microbolometer-based un-cooled IR detectors without using ROIC, Sensor. Actuat. A-
Phys., 141, 359-366, 2008.

Schieferdecker, J., Quad, R., Holzenkämpfer, E., and Schulze, M.: Infrared thermopile sensors with high sensitivity and very low temperature coefficient, Sensor. Actuat. A-Phys., 46-47, 422427, 1995.

Schnelle, W., Dillner, U., Scheike, B., and Albrecht, L.: Infrared radiation sensors based on thin film bolometers, Proceedings of the 2nd Symposium on Temperature Measurement in Industry and Science, Suhl, Germany, 16-18 October 1984, 195-204, 1984.

Smith, R. A., Jones, F. E., and Chasmar, R. P.: The detection and measurement of infra-red radiation, Clarendon Press, Oxford, 1968.

Tanaka, A., Matsumoto, S., Tsukamoto, N., Itoh, S., Chiba, K., Endoh, T., Nakazato, A., Okuyama, K., Kumazawa, Y., Hijikawa, M., Gotoh, H., Tanaka, T., and Teranishi, N.: Infrared focal plane array incorporating silicon IC process compatible bolometer, IEEE T. Electron Dev., 43, 1844-1850, 1996.

Tissot, J. L., Trouilleau, C., Fieque, B., Crastes, A., and Legras, O.: Uncooled microbolometer detector recent developments at Ulis, Proceedings of SPIE 5957, Infrared Photoelectronics, Warsaw, Poland, 30-31 August 2005, 59570M1-12, 2005.

Tissot, J. L., Durand, A., Garret, T., Minassian, C., Robert, P., Tinnes, S., and Vilain, M.: High performance uncooled amorphous silicon VGA IRFPA with $17 \mu \mathrm{m}$ pixel-pitch, Proceedings of SPIE 7660, Infrared Technology and Applications XXXVI, Orlando, USA, 5-9 April 2010, 76600T1-7, 2010.

van Herwaarden, A. W. and van Oudheusden, B.: Thermal sensors, edited by: Meijer, G. C. M. and van Herwaarden, A. W., Institute of Physics Publishing, Bristol and Philadelphia, 1994.

Venkatasubramanian, R., Siivola, E., Colpitts, T., and O’Quinn, B.: Thin-film thermoelectric devices with high room-temperature figures of merit, Nature, 413, 597-602, 2001.

Vera-Reveles, G., Simmons, T. J., Bravo-Sánchez, M., Vidal, M. A., Navarro-Contreras, H., and González, J.: High-sensitivity bolometers from self-oriented single-walled carbon nanotube composites, ACS Appl. Mater. Interfaces, 3, 3200-3204, 2011.

Völklein, F., Wiegand, A., and Baier, V.: High-sensitivity radiation thermopiles made of Bi-Sb-Te films, Sensor. Actuat. A-Phys., 29, 87-91, 1991

Wang, K., Xue, C., Liang, T., Jiao, B., Zhang, W., Chen, D., and Xiong, J.: Thermopile infrared detector with detectivity greater than $10^{8} \mathrm{cmHz}^{1 / 2} / \mathrm{W}$, J. Infrared Millim. Te., 31, 810-820, 2010.

Wang, L.-X. and Li, X.-A.: Preparation of $\mathrm{VO}_{2}$ microbolometer for $\mathrm{CO}_{2}$ gas detection, Proceedings of the 2010 International Conference on Microwave and Millimeter Wave Technology, Chengdu, China, 8-11 May 2010, 1774-1777, 2010.

Wood, R. A.: Uncooled thermal imaging with monolithic silicon focal planes, Proceedings of SPIE 2020, Infrared Technology XIX, San Diego, USA, 11 July 1993, 322-329, 1993.

Yue, R.-F., Dong, L., and Liu, L.-T.: Integrated a-Si:B microbolometer arrays based on improved porous silicon micromachining techniques, Chinese Phys. Lett., 23, 1331-1334, 2006.

Zintu, D., Tosone, G., and Mercuri, A.: Dual ion beam sputtering vanadium dioxide microbolometers by surface micromachining, Infrared Phys. Techn., 43, 245-250, 2002. 